\title{
Does grip strength predict squat strength in healthy young men?
}

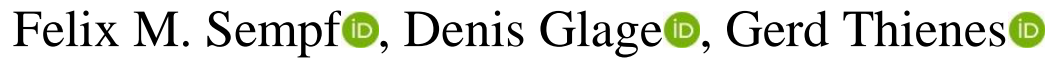 \\ Institute for Sport Science, Georg-August-University Göttingen, Göttingen, Germany.
}

\begin{abstract}
Squat repetition maximum (RM) testing is a reliable and safe method for assessing lower body strength in healthy individuals but constraints in technique, time or access to testing devices can restrict its application. Measuring grip strength may provide a simple alternative as it has been linked to lower body strength in non-athletes. However, to date no study has examined the relationship between grip strength and squat strength in an untrained population. Therefore, the purpose of this study was to investigate the predictability of squat performance by grip strength. 25 untrained healthy male adults $(24.9 \pm 3.4$ years) were tested for maximal grip strength and squat 5RM, which was used to calculate the estimated squat 1RM. Our data revealed a significant and strong correlation between grip strength and estimated squat 1RM ( $\mathrm{r}=$ $.68, \mathrm{p}<.001)$ as well as a significant regression equation in which grip strength can explain $46 \%$ of estimated squat $1 \mathrm{RM}\left(\mathrm{r}^{2}=.46, \mathrm{p}<.001\right)$. The results suggest that grip strength may be used to predict squat performance in untrained healthy male adults, when repetition maximum testing is not feasible.
\end{abstract}

Keywords. Dynamometer, grip, repetition maximum, squat.

\section{Introduction}

Muscle strength is an important determinant of health and physical fitness. Increasing muscle strength can reduce all-cause as well as cardiovascular mortality risk (Artero et al., 2012; Kim et al., 2018) and lower the risk of osteoporosis in both men and women (McGrath et al., 2017). Maintaining strength in old age has been associated with lower prevalence rates of functional limitations in activities of daily living, such as walking or climbing stairs, as well as a reduced rate of falls (Brill et al., 2000; Kell et al., 2001; Vasconcelos Rocha et al., 2016; Wind et al., 2010). Lower body strength in particular is linked to the successful completion of daily activities in the elderly (Puthoff \& Nielsen, 2007). Consequently, it is recommended to perform strength training already at an early age and maintain strength throughout the lifespan (Faigenbaum, 2000; Kell et al., 2001; Westcott, 2012). In this context, the squat is considered a good exercise to increase general health and fitness due to its ability to mimic daily tasks and effectiveness in increasing entire lower limb and overall body strength (Kraemer et al., 2002; Myer et al., 2014; Vecchio et al., 2018). Squat strength may also be of importance as it has been associated with muscular power (Seitz et al., 2014; Wirth et al., 2016) which has recently been linked to mortality in middle-aged and old adults (Araujo et al., 2019). Therefore, identifying people with below-average squat performance may help to prevent the age-related loss of muscle function that begins in the fourth decade of life and delay the onset of frailty at a later age. For strength testing under nonlaboratory conditions, repetition maximum (RM) protocols are considered the gold standard (Levinger et al., 2009) and the validity and reliability has been established for the squat (Seo et al., 2012; Urquhart et al., 2015). However, insufficient technique, time con- 
straints or the lack of adequate testing devices may be contraindications for the safe completion of RM testing in this exercise. Consequently, there is a need for quick and feasible methods to assess squat strength, particularly under field conditions. Among these, measuring grip strength using a handheld dynamometer is a promising alternative. Although initially being used for assessing hand and forearm strength (Nwuga, 1975), studies have established its association with strength in diverse subpopulations, such as children, adolescents, adults, elders (Bohannon, 2019; Debeliso et al., 2015a; Debeliso et al., 2015b; Matsudo et al., 2015; Trosclair et al., 2011; Wind et al., 2010), tactical professionals (Rhea et al., 2004) and athletes (Drid et al., 2015; Jawan et al., 2014; Zampagni et al., 2008). While grip strength appears to be a good indicator for overall strength in nonathletic populations, to our knowledge no study thus far has assessed the relationship between squat strength and grip strength. Therefore, the purpose of this study was to examine the relationship of these variables in healthy male adults with little prior strength training experience and no athletic background. In addition, the predictability of squat strength by grip strength was assessed. Based on previous research, it was hypothesized that grip strength is associated with squat strength and that it can serve as a predictor of squat strength in healthy but untrained young men.

\section{Methods}

Using a descriptive study design, we examined the association between grip strength and squat strength. Grip strength 1RM and squat 5RM were assessed under the direct supervision of a trained sport scientist. All data collection took place within a week and consisted of a familiarization session and the actual assessment.

\section{Subjects}

An a priori power analysis using $\mathrm{G}^{*}$ Power (Faul et al., 2007) indicated that a sample size of 29 would be sufficient to detect a significant medium interaction effect $(\mathrm{d}=.50)$ with a power of .85 and an alpha of .05 . Therefore, 30 active healthy male subjects were recruited for this study. All subjects had the risks and benefits explained to them beforehand and signed an institutionally approved consent form to participate. The study was designed according to the latest version of the Declaration of Helsinki and approved by the local ethics committee of the Georg-AugustUniversity Göttingen. Exclusion criteria consisted of acute and unhealed musculoskeletal injuries, as well as pre-existing cardiovascular or metabolic diseases.

\section{Procedures}

A week before the squat test, a familiarization session for the exercise technique took place with all participants. Key instructions for the squat technique were as follows: Barbell placed across the upper back, feet positioned shoulder-width apart and slightly rotated outwards, keeping a neutral spine while performing the exercise all the time, no valgus at the knees and a squat depth to the point where the femurs are slightly past parallel to the ground (Myer et al., 2014). On the day of the experiment, participants completed a general warmup for five minutes on a cross-trainer (Nautilus E 916) with low to moderate intensity (Haff \& Triplett, 2016) at a heart rate of approximately $120-140$ beats per minute. Participants were subsequently tested for their maximum grip strength on the dominant hand (Agbuga et al., 2009) with a Jamar handgrip dynamometer (Baseline Evaluation Instruments). Testing was conducted according to standardized guidelines (Roberts et al., 2011): Participants stood with their feet shoulder-width apart, shoulders adducted and neutrally rotated, elbow flexed at $90^{\circ}$, forearm in neutral position. After one familiarization trial, three further attempts were carried out 
and the achieved maximum score was used for the analysis. Between each trial, the subjects rested for 30 seconds. A rest period of two minutes followed the grip strength measurement.

A 5RM protocol was used to estimate squat 1RM. To ensure proper exercise technique and safety of the participants, trained sports scientists supervised all tests, and an instructor to participants' ratio of 1:1 was maintained. The participants did a specific warmup for the squat 5RM test by completing one set of 8-10 repetitions with an empty barbell and three additional warm-up sets with five repetitions. These sets were performed with added weights, which was determined under the premise of proper technique and effort at 60, 75 and $90 \%$ 5RM. Perceived exertion rated on a Borg scale was used to estimate subsequent weight increments (Foster et al., 2001). Rest periods were two minutes between warm-up sets. Subsequently, participants were asked to squat their estimated 5RM weight. If the predefined criteria were met and an attempt was rated as submaximal, $5 \mathrm{~kg}$ was added to the bar and another set was carried out after an extended rest period of five minutes. In case of improper technique or failure in the exercise, the execution was stopped and the properly performed repetitions were counted. If a participant rated a set as maximal, no more sets were conducted. The maximal weights and number of successfully completed repetitions of the last set were used to calculate estimated 1RM using Epley's Formula (DiStasio, 2014).

\section{Statistical Analyses}

All statistical analysis was performed using SPSS 25 . Normality of data was assessed by the
Shapiro-Wilk test, which determined all primary outcome measures of interest to be normally distributed. Inclusion criteria of the participants for the statistical analysis was a strength level below intermediate. Therefore, depending on the actual weight class of each individual, participants with a bodyweight ratio $\geq 1.58-1.61$ (Clark, 2020) in squat strength were excluded. The relationship between grip strength and the estimated squat 1RM was computed using Pearson's r. A simple linear regression was calculated to predict estimated squat 1RM based on grip strength. The alpha level for significance for all statistical tests was set at $\mathrm{p}<.05$.

\section{Results}

Five participants were excluded due to their intermediate squat strength so that a total of 25 participants remained. Anthropometric data of the final sample are listed in Table 1, average grip strength, estimated squat $1 R M$ and estimated squat 1RM in relation to body mass in Table 2.

Grip strength and estimated squat 1RM showed a strong and significant correlation, while grip strength and squat strength in relation to body mass did not, see Table 3.

A significant linear regression equation was found $\left(\mathrm{F}_{(1.23)}=19.777, p<.001, \mathrm{r}^{2}=.46\right)$ : Participants predicted estimated squat $1 \mathrm{RM}$ is equal to $40.269+1.109 *$ grip strength when estimated squat 1RM is measured in $\mathrm{kg}$. Estimated squat 1RM increased for 1.109 for each $\mathrm{kg}$ of grip strength. The regression line is provided in the scatter plot.

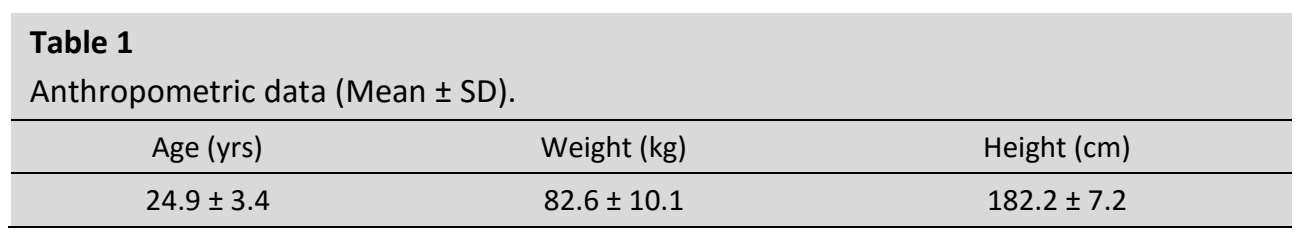




\begin{tabular}{|c|c|c|}
\hline $\begin{array}{l}\text { Table } 2 \\
\text { Performance variab }\end{array}$ & Mean \pm SD) & \\
\hline Grip Strength (kg) & Estimated Squat 1RM (kg) & Estimated Squat 1RM/Body Mass \\
\hline $52.9 \pm 9.1$ & $98.9 \pm 14.9$ & $1.21 \pm 0.2$ \\
\hline
\end{tabular}

\section{Table 3}

Pearson Correlation Coefficients ( $r$ ) between grip strength and squat strength / in relation to body mass)

\begin{tabular}{lcc} 
& Estimated Squat 1RM $(\mathrm{kg})$ & Estimated Squat 1RM/ Body Mass \\
\hline Grip Strength $(\mathrm{kg})$ & $.68^{*}$ & .384 \\
\hline${ }^{*} p<.01$ & &
\end{tabular}

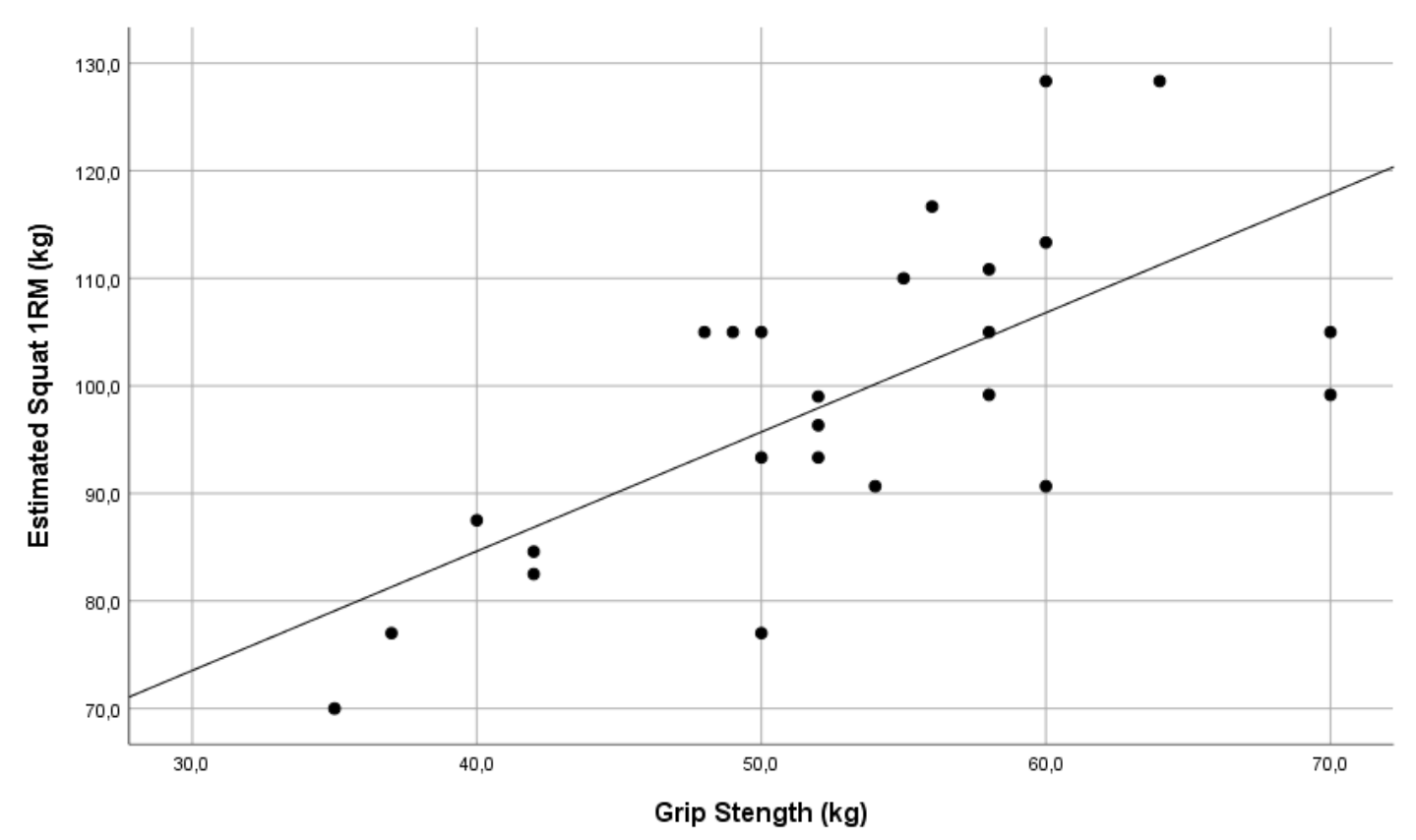

Figure 1. Scatter plot of the correlation between grip strength and estimated squat 1RM.

\section{Discussion}

The purpose of this study was to examine the relationship between squat strength and grip strength and investigate the predictability of squat strength by grip strength in healthy male adults with little or no prior weight training experience. 5RM testing is a valid predictor of 1RM (Dohoney et al., 2002; Reynolds et al., 2006) and has been used in this study because of its lower risk for injury compared to $1 \mathrm{RM}$ testing in untrained populations (Gail et al., 2015; Haff \& Triplett, 2016; Levinger et al., 2009). The mean grip strength reported is in accordance with normative values (Beck \& Bös,
1995) and the mean estimated squat $1 R M$ in relation to body mass is in the range for novices as reported by Clark (2020). With respect to our research question, we found a strong correlation between grip strength and squat strength and a linear regression with a large effect size (Döring \& Bortz, 2016). These results suggest that grip strength is a valid indicator of squat performance and, following our reasoning, lower body strength in this population. Our findings are in agreement with previous research by Trosclair et al. (2011) who observed similar correlations of grip strength with leg-press $(r=.72)$ and legextension $(r=.61) 1 R M$ in a study with college- 
aged students. Grip strength therefore seems to be correlated to both functional and lessfunctional lower body strength in young untrained male adults, likewise. In contrast to these observations in untrained populations, a study by Otterson \& Debeliso (2020) found no significant correlation between squat performance and grip strength in football athletes. Earlier research by Jawan (2014) and Agbuga (2009) supports these results for (team) sport athletes as they did not observe any significant correlations of grip strength with various upper and lower body 1RM tests. Grip strength showed also no significant correlation with anerobic fitness or non-gripping bodyweight movements, with the exception of sit-ups, in female crossfit athletes (Haynes \& Debeliso, 2019). The lack of correlation between grip strength with general and lower body strength in athletes, as well as nongripping tasks and movements, may be explained by the specificity principle of training, which states that induced changes are specific to the exercise stress (Enoka, 1998). It is likely, that athletes strengthen commonly tested muscles like the $\mathrm{m}$. quadriceps femoris through their sports and/or additional strength training. Consequently, higher general strengths levels occur but grip strength is not increased equally due to lack of an equal training stimulus. In non-athletes however, muscles are developed on a similar untrained level, which allows grip strength to represent the general ability to exert tension throughout the body. Hence, grip strength can be used as an indicator for overall and lower body strength in untrained populations but not for athletes from team sports. However, the most accurate way to assess lower body strength in non-laboratory conditions remains by testing for the 1RM in exercises like the squat (Seo et al., 2012).

Major limitations of our study are the small sample size and the lack of assessing participants' backgrounds regarding regular tasks and activities that affect grip strength specifically, i.e. climbing. This may partly explain squat underperformance in our sample. Consequently, further studies should use larger sample sizes and screen future participants for recreational activities that may be related to grip strength.

\section{Conclusion}

Our results indicate that grip strength can be used as an indicator for squat strength in healthy young adults without the risk of fatiguing or injury and with minimal requirement in equipment and time. Therefore, health practitioners and coaches may use grip strength to assess squat strength in healthy, yet untrained male adults, when repetition maximum testing is not feasible.

\section{Acknowledgements}

The authors declare that there is no conflict of interest.

\section{References}

Agbuga, B., Konukman, F., \& Yilmaz, I. (2009). Prediction of upper body strength by using grip strength test in Division II American college football players' grip strength. Hacettepe J of Sport Sciences, (20), 16-23.

Araujo, C.G., Castro, C.L., Franca, J.F., Laukkanen, J.A., Hamar, D., \& Myers, J. (2019). Muscle power in upright row movement: predictor of all-cause mortality in individuals between 41 and 85 years of age: preliminary results. Eur $\mathrm{J}$ Prev Cardiol, 26(6, Suppl.), 459.

Artero, E.G., $\quad$ Lee, D.-c., $\quad$ Lavie, C.J., $\quad$ EspañaRomero, V., Sui, X., Church, T.S., \& Blair, S.N. (2012). Effects of muscular strength on cardiovascular risk factors and prognosis. $J$ Cardiopulm Rehabil Prev, 32(6), 351-358.

Beck, J., \& Bös, K. (1995). Normwerte motorischer Leistungsfähigkeit (1. Aufl.). Bundesinstitut für Sportwissenschaft: Vol. 95,5. Köln: Sport u. Buch Strauß Ed. Sport.

Bohannon, R.W. (2019). Grip strength: an indispensable biomarker for older adults. Clin Interv Aging, 14, 1681-1691. 
Brill, P.A., Macera, C.A., Davis, D.R., Blair, S.N., \& Gordon, N. (2000). Muscular strength and physical function. Med Sci Sports Exerc, 32(2), 412-416.

Clark, M. (2020). Squat Standards. Retrieved from https://strengthlevel.com/strength-

standards/squat

DeBeliso, M., Boham, M.D., Harris, C.G., Carson, C., Berning, J.M., Sevene, T.G., \& Adams, K.J. (2015a). Grip and body strength measures in the mature adult: a brief report. International Journal of Science and Engineering Investigations, 4(37), 8386.

DeBeliso, M., Boham, M.D., Harris, C.G., Carson, C., Berning, J.M., Sevene, T.G., Adams, K.J., Climstein, M. (2015b). Grip strength and functional measures in the mature adult: brief report II. International Journal of Science and Engineering Investigations, 4(39), 1-4.

DiStasio, T.J. (2014). Validation of the Brzycki and Epley Equations for the 1 Repetition Maximum Back Squat Test in Division I College Football Players. Retrieved from https://opensiuc.lib.siu.edu/cgi/viewcontent.cgi? article $=1744 \&$ context $=$ gs_rp

Dohoney, P., Chromiak, J., Lemire, D., Abadie, B., \& Kovacs, C. (2002). Prediction of one repetition maximum (1-RM) strength from a 4-6 RM and a 7-10 RM submaximal strength test in healthy young adult males. J Exerc Physiol Online, (5), 5459.

Döring, N., \& Bortz, J. (2016). Forschungsmethoden und Evaluation in den Sozial- und Humanwissenschaften (5. vollständig überarbeitete, aktualisierte und erweiterte Auflage). Springer-Lehrbuch. Berlin, Heidelberg: Springer.

Drid, P., Casals, C., Mekic, A., Radjo, I., Stojanovic, M., \& Ostojic, S.M. (2015). Fitness and anthropometric profiles of international vs. national judo medalists in half-heavyweight category. J Strength Cond Res, 29(8), 2115-2121.

Enoka, R. M. (1998). Neuromechanical basis of kinesiology (2. ed.). Champaign, Ill.: Human Kinetics.

Faigenbaum, A.D. (2000). Strength training for children and adolescents. Clin Sports Med, 19(4), 593-619.
Faul, F., Erdfelder, E., Lang, A.-G., \& Buchner, A. (2007). G* power 3: A flexible statistical power analysis program for the social, behavioral, and biomedical sciences. Behav Res Methods, 39(2), 175-191.

Foster, C., Florhaug, J.A., Franklin, J., Gottschall, L., Hrovatin, L.A., Parker, S., Doleshal, P., \& Dodge, C. (2001). A new approach to monitoring exercise training. J Strength Cond Res, 15(1), 109115.

Gail, S., Argauer, P., \& Künzell, S. (2015). Validität eines 5-RM Krafttests im Gesundheits- und Fitnesssport. Swiss Sports $\mathcal{E}$ Exercise Medicine, 63(3).

Haff, G.G., \& Triplett, N.T. (Eds.) (2016). Essentials of strength training and conditioning (4th edition). Champaign, IL, Windsor, ON, Leeds: Human Kinetics.

Haynes, E., \& Debeliso, M. (2019). The relationship between CrossFit performance and grip strength. Turk J Kinesiol, 5(1), 15-21.

Jawan, L., Adnan, R., Sulaiman, N., \& Ismail, S.I. (2014). Efficacy of handgrip strength in predicting total body strength among high performance athletes. In R. Adnan, S.I. Ismail, \& N. Sulaiman (Eds.), Proceedings of the International Colloquium on Sports Science, Exercise, Engineering and Technology (ICOSSEET 2014) (pp. 29-38). Singapore: Springer.

Kell, R.T., Bell, G., \& Quinney, A. (2001). Musculoskeletal fitness, health outcomes and quality of life. Sports Medicine (Auckland, N.Z.), 31(12), 863-873.

Kim, Y., White, T., Wijndaele, K., Westgate, K., Sharp, S.J., Helge, J.W., Wareham, N.J., \& Brage, S. (2018). The combination of cardiorespiratory fitness and muscle strength, and mortality risk. Eur J Epidemiol, 33(10), 953964.

Kraemer, W.J., Adams, K., Cafarelli, E., Dudley, G. A., Dooly, C., Feigenbaum, M.S., .. . TriplettMcBride, T. (2002). American College of Sports Medicine position stand. Progression models in resistance training for healthy adults. Med Sci Sports Exerc, 34(2), 364-380.

Levinger, I., Goodman, C., Hare, D.L., Jerums, G., Toia, D., \& Selig, S. (2009). The reliability of the 
1RM strength test for untrained middle-aged individuals. J Sci Med Sport, 12(2), 310-316.

Matsudo, V.K.R., Matsudo, S.M., Rezende, L.F. M.d., \& Raso, V. (2015). Força de preensão manual como preditor de aptidão física em crianças e adolescentes. Rev Bras Cineantropom Desempenho Hum, 17(1), 1.

McGrath, R.P., Kraemer, W.J., Vincent, B.M., Hall, O.T., \& Peterson, M.D. (2017). Muscle Strength Is Protective Against Osteoporosis in an Ethnically Diverse Sample of Adults. J Strength Cond Res, 31(9), 2586-2589.

Myer, G.D., Kushner, A.M., Brent, J.L., Schoenfeld, B.J., Hugentobler, J., Lloyd, R.S., Vermeil, A., Chu, D.A., Harbin J., McGill, S.M. (2014). The back squat: a proposed assessment of functional deficits and technical factors that limit performance. Strength Cond J, 36(6), 4-27.

Nwuga, V.C. (1975). Grip strength and grip endurance in physical therapy students. Arch Phys Med Rehabil, 56(7), 297-300.

Otterson, R., \& Debeliso, M. (2020). Grip Strength and North American Collegiate Football Performance Indicators. Turk J Kinesiol, 6(1), 1625.

Puthoff, M.L., \& Nielsen, D.H. (2007). Relationships among impairments in lower-extremity strength and power, functional limitations, and disability in older adults. Physical Therapy, 87(10), 13341347.

Reynolds, J.M., Gordon, T.J., \& Robergs, R.A. (2006). Prediction of one repetition maximum strength from multiple repetition maximum testing and anthropometry. J Strength Cond Res 20(3), 584592.

Rhea, M.R., Alvar, B.A., \& Gray, R. (2004). Physical fitness and job performance of firefighters. $J$ Strength Cond Res, 18(2), 348-352.

Roberts, H.C., Denison, H.J., Martin, H.J., Patel, H.P., Syddall, H., Cooper, C., \& Sayer, A.A. (2011). A review of the measurement of grip strength in clinical and epidemiological studies: towards a standardised approach. Age and Ageing, 40(4), 423-429.

Seitz, L.B., Reyes, A., Tran, T.T., Saez de Villarreal, E., \& Haff, G.G. (2014). Increases in lower-body strength transfer positively to sprint performance: A systematic review with metaanalysis. Sports Medicine (Auckland, N.Z.), 44(12), 1693-1702.

Seo, D.-I., Kim, E., Fahs, C.A., Rossow, L., Young, K., Ferguson, S.L.,... So, W.-Y. (2012). Reliability of the one-repetition maximum test based on muscle group and gender. I Sci Med Sport, 11(2), 221-225.

Trosclair, D., Bellar, D., Judge, L.W., Smith, J., Mazerat, N., \& Brignac, A. (2011). Hand-grip strength as a predictor of muscular strength and endurance. J Strength Cond Res, 25(Suppl. 1), S99.

Urquhart, B.G., Moir, G.L., Graham, S.M., ～\& Connaboy, C. (2015). Reliability of 1RM splitsquat performance and the efficacy of assessing both bilateral squat and split-squat 1RM in a single session for non-resistance-trained recreationally active men. J Strength Cond Res, 29(7), 1991-1998.

Vasconcelos Rocha, S., Souza dos Santos, S., Carneiro Vasconcelos, L.R., \& Alves dos Santos, C. (2016). Strength and ability to implement the activities of daily living in elderly resident in rural areas. Colombia Médica: CM, 47(3), 167-171.

Vecchio, L.D., Daewoud, H., \& Green, S. (2018). The health and performance benefits of the squat, deadlift, and bench press. MOJ Yoga \& Physical Therapy, 3(2), 40-47.

Westcott, W.L. (2012). Resistance training is medicine: Effects of strength training on health. Curr Sports Med Rep, 11(4), 209-216.

Wind, A.E., Takken, T., Helders, P.J.M., \& Engelbert, R.H.H. (2010). Is grip strength a predictor for total muscle strength in healthy children, adolescents, and young adults? Eur J Pediatr, 169(3), 281-287.

Wirth, K., Hartmann, H., Sander, A., Mickel, C., Szilvas, E., \& Keiner, M. (2016). The impact of back squat and leg-press exercises on maximal strength and speed-strength parameters. J Strength Cond Res, 30(5), 1205-1212.

Zampagni, M.L., Casino, D., Benelli, P., Visani, A., Marcacci, M., De Vito, G. (2008). Anthropometric and strength variables to predict freestyle performance times in elite master swimmers. $J$ Strength Cond Res, 22(4), 1298-1307. 